\title{
Trinkwasserbefunde - Auswertung langjähriger Daten der Trinkwassertemperatur und mikrobiologischer Parameter mit Indikatorfunktion
}

\author{
Christoph Schönher · David Kerschbaumer · Philipp Proksch · Reinhard Perfler
}

Angenommen: 1. September 2020 / Online publiziert: 29. September 2020

(C) Der/die Autor(en) 2020

Zusammenfassung Die Bereitstellung von mikrobiologisch sicherem Trinkwasser ist eine der Hauptaufgaben der Trinkwasserversorgungsinfrastruktur. Regelmäßige Trinkwasseruntersuchungen sind ein wesentlicher Garant zur Einhaltung hygienischer Anforderungen und können in der Zusammenschau Hinweise auf längerfristige Entwicklungen und generelle Zusammenhänge liefern. Im Rahmen eines Projekts zur Ermittlung des Temperatureinflusses in der Trinkwasserversorgung wurde vom Land Niederösterreich ein anonymisierter Auszug der Befunddatenbank zur Auswertung übermittelt. Der wesentliche Fokus liegt dementsprechend auf der Analyse der Temperaturmessungen im Zusammenhang mit den mikrobiologischen Parameter KBE bei $22^{\circ} \mathrm{C}, 37^{\circ} \mathrm{C}$ und den coliformen Bakterien. Zudem wurden die meisten Auswertungen für den Bereich der Trinkwasserverteilung vorgenommen.

Hinsichtlich langfristiger Trends zeigen die Daten eine jährliche Zunahme der gemessenen Temperatur bei Netzprobenahmen von etwa $0,064^{\circ} \mathrm{C}$. Perioden mit sehr hohen Werten $\left(>20^{\circ} \mathrm{C}\right)$ der Wassertemperatur wurden im $\mathrm{Au}$ gust 2015 und 2018 verzeichnet (jede dritte Netzprobe). Überwiegend abnehmende Trends wurden für die mikrobiologischen Parameter ermittelt. Für die $\mathrm{KBE}$ bei $22^{\circ} \mathrm{C}$ beträgt der Rückgang etwa $0,5 \mathrm{KBE} / \mathrm{ml}$ pro Jahr. Trotz dieser gegenläufigen Tendenzen ist nicht davon auszugehen, dass die Zunahme

DI C. Schönher $(\bowtie)$

DI D. Kerschbaumer · DI P. Proksch •

PD DI Dr. R. Perfler

Department

Wasser-Atmosphäre-Umwelt,

Institut für Siedlungswasserbau, Industriewasserwirtschaft

und Gewässerschutz (SIG),

Universität für Bodenkultur Wien,

Muthgasse 18, 1190 Wien, Österreich

christoph.schoenher@boku.ac.at der Temperaturen keine (negativen) Auswirkungen auf die mikrobiologische Situation haben kann. Im Rahmen einer Regressionsanalyse wurde der Einfluss der Temperatur für die $\mathrm{KBE}$ bei $37^{\circ} \mathrm{C}$ als signifikant ermittelt. So erhöht eine Zunahme der Temperatur von $15^{\circ} \mathrm{C}$ auf $20^{\circ} \mathrm{C}$ die Wahrscheinlichkeit von Werten $>20 \mathrm{KBE} / \mathrm{ml}$ von 0,077 auf 0,109 . Dieser Befund ist aus mikrobiologischer Hinsicht als plausibel zu bezeichnen, wenngleich die Temperatur hier auch lediglich längere Netzaufenthaltszeiten anzeigen könnte. Der Einfluss weiterer Faktoren, etwa des Organik-Gehalts im Wasser, kann aufgrund der Datenlage weniger gut abgebildet werden, ist aber sicher als ebenso wichtig zu bezeichnen.

Schlüsselwörter Wasserversorgung Trinkwasserbefund · Mikrobiologie . Temperatur · Datenauswertung · Trend $\cdot$ Regressionsmodell

\section{Drinking water}

testings - evaluation of long-term data of temperature and microbiological parameters

Abstract The provision of microbiologically safe drinking water is one of the main tasks of drinking water supply. Regular drinking water tests are an essential guarantee for the compliance with hygienic requirements and can provide information on long-term developments and general correlations between parameters. Within the scope of a project to determine the temperature influence in the drinking water supply, authorities from Lower Austria made an anonymized selection of their database available for evaluation. Accordingly, the main focus is on the analysis of temperature measurements in connection with the microbiological parameters $\mathrm{CFU}$ at $22^{\circ} \mathrm{C}, 37^{\circ} \mathrm{C}$ and the coliform bacteria. In addition, most of the evaluations were carried out for the drinking water distribution system.

With regard to long-term trends, the data show an annual increase of the measured temperature at sampling points within in the distribution network of about $0.064^{\circ} \mathrm{C}$. Periods with very high values of water temperature were recorded in August 2015 and 2018 as every third sample in the distribution network exceeded $20^{\circ} \mathrm{C}$. Mainly decreasing trends were found for the microbiological parameters. For CFU at $22^{\circ} \mathrm{C}$ the decrease is about $0.5 \mathrm{CFU} / \mathrm{ml}$ per year. Despite these contrary trends, it cannot be assumed that the increase in temperature will not have a (negative) impact on the microbiological situation. In a regression analysis, the influence of temperature was found to be significant for the $\mathrm{CFU}$ at $37^{\circ} \mathrm{C}$. Thus, an increase in temperature from $15^{\circ} \mathrm{C}$ to $20^{\circ} \mathrm{C}$ increases the probability of values $>20 \mathrm{CFU} / \mathrm{ml}$ from 0.077 to 0.109 . This finding can be considered plausible from a microbiological point of view, although the temperature here could only indicate longer network residence times. The influence of other at least equally important factors, such as the organic content of the water, can't be represented as precisely due to a lack of available data.

Keywords Water supply · Drinking water testing - Microbiology .

Temperature $\cdot$ Data evaluation · Trend . Regression analysis

\section{Einleitung}

Der Zugang zu sauberem Trinkwasser ist von umfassender Wichtigkeit. Das Wasser muss dabei geeignet sein, ohne Gefährdung der menschlichen Gesundheit getrunken oder verwendet zu werden. Die Bereitstellung von mikrobiologisch sicherem Trinkwasser ist eine der Hauptaufgaben der Trinkwasserversorgungsinfrastruktur und die 
Überwachung des Trinkwassers von der Quelle bis zum Wasserhahn ein wesentlicher Schritt zur Einhaltung hygienischer Anforderungen.

Die klimatischen Veränderungen der letzten Jahrzehnte zeigen sich unter anderem in einem Anstieg der Lufttemperaturen. Infolge dieser Änderungen kommt es auch zu einer Änderung des Temperaturhaushalts im Untergrund. Betroffen davon sind ressourcenseitig gerade oberflächennahe Grundwasserkörper und von einer weiteren generellen Zunahme ist auszugehen (Blöschl et al. 2017). Die Temperaturänderungen ziehen mikrobiologische und physikalisch-chemische Änderungen nach sich. So konnte etwa bereits gezeigt werden, dass die Temperaturzunahmen mit einer Abnahme der Sauerstoffsättigung verbunden sind (Riedel 2019). Ein besonders betroffener Bereich der Temperaturzunahmen ist die Trinkwasserverteilung. In Abhängigkeit von Oberflächen- und Untergrundbeschaffenheit, Verlegetiefe und Betriebsweisen können sehr hohe Temperaturen im Verteilnetz erreicht werden.

Trinkwasser in öffentlichen Trinkwasserverteilungssystemen enthält immer Mikroorganismen, einerseits frei im Trinkwasser, andererseits in Form von Biofilmen auf allen mit Trinkwasser in Kontakt stehenden Oberflächen. Physikalische, chemische und biologische Faktoren sind für das Überleben und das Wachstum von Bakterien (Vermehrung) in der Umwelt entscheidend. In der Trinkwasserverteilung sind hier die Wassertemperatur, die Nährstoffverhältnisse, die hydraulischen Bedingungen sowie die Anwesenheit von Desinfektionsmitteln von Bedeutung (Prest et al. 2016). Kurzfristig auftretende (Hitzewellen) und saisonale Änderungen der Temperaturen wirken sich auf die mikrobiellen Prozesse und die Diversität der mikrobiellen Gemeinschaften in Wassersystemen aus. Verschiedene Bakterienarten zeichnen sich durch oftmals deutlich unterschiedliche optimale Wachstumstemperaturen aus. Im Trinkwasser sind hauptsächlich mesophile Bakterien von hygienischer Relevanz, deren Optimum im Bereich zwischen $25^{\circ} \mathrm{C}$ und $40^{\circ} \mathrm{C}$ liegt (Grobe et al. 2014).

Nach Vorgabe der österreichischen Trinkwasserverordnung besteht für Wasserversorger die Pflicht zur regelmäßigen Untersuchung des Trinkwassers in mikrobiologischer und chemisch-physikalischer Hinsicht. In mi- krobiologischer Hinsicht werden dabei unter anderen die Parameter koloniebildende Einheiten (KBE pro $\mathrm{ml}$ ) bei $22^{\circ} \mathrm{C}$ und $37^{\circ} \mathrm{C}$ Bebrütungstemperatur nach EN ISO 6222 (Plattengussverfahren) sowie coliforme Bakterien (Coliforme pro 100 bzw. $250 \mathrm{ml}$ ) nach EN ISO 9308-1 (Membranfiltration) oder EN ISO 9308-2 (MPN-Verfahren) erfasst. Die Bestimmung der KBE dient einer allgemeinen Erfassung mikrobiologischer Veränderungen und kann etwa Hinweise auf Verunreinigungen des Wassers nach der Aufbereitung oder lange Stagnationszeiten liefern. Die Bebrütung bei verschiedenen Temperaturen soll das auftretende Keimspektrum erweitern, wobei $37^{\circ} \mathrm{C}$ in etwa der menschlichen Körpertemperatur entsprechen. Coliforme Bakterien sind eine sehr heterogene Gruppe von Enterobakterien, die auch Gattungen umfassen, die außerhalb des Darmtrakts vorkommen und damit keine Indikatoren für eine fäkale Verunreinigung darstellen (Feuerpfeil und Botzenhart 2008). Das Auftreten von Coliformen kann verschiedene Ursachen haben und muss nicht immer auf einen (aktuellen) Eintrag von außerhalb hindeuten (Biofilme, Mobilisierung aus Ablagerungen) (UBA 2009). Für die genannten mikrobiologischen Parameter - auch für die KBE - gilt jedenfalls, dass nur ein sehr geringer Teil aller im Wasser vorkommenden und vermehrungsfähigen Bakterien erfasst wird (Van Nevel et al. 2017).

Die Befunde aus den Trinkwasseruntersuchungen werden auf Landesebene von den Behörden erfasst. Im Rahmen einer aktuellen Studie zum Einfluss von Temperaturerhöhung auf die Trinkwasserversorgung wurde ein umfangreicher Datenbestand aus Niederösterreich in anonymisierter Form übermittelt, der neben den genannten mikrobiologischen Parametern auch physikalischchemische Parameter (Temperatur, Gesamthärte etc.) und betriebliche Informationen (Vorhandensein einer Desinfektionsmaßnahme) enthält. Ziel der vorliegenden Auswertung ist es, anhand dieses Datenbestands Charakteristika (Trends bzw. jährliche Verteilung) der mikrobiologischen Parameter und der Temperatur zu beleuchten sowie ausgewählte Einflussfaktoren auf die mikrobiologischen Parameter modellhaft zu erfassen. Das Hauptaugenmerk liegt dabei auf Untersuchung im Bereich der Trinkwasserverteilung. Ausführlichere Darstellungen unter Einbeziehung wei- terer Datenquellen werden im Bericht zur erwähnten Studie („Auswirkungen erhöhter Wassertemperaturen bei der Trinkwassergewinnung, -speicherung und -verteilung“) zugänglich gemacht.

\section{Material und Methoden}

Alle Auswertungen wurden mit der frei verfügbaren Programmiersprache „R“ durchgeführt (R-Core Team 2019). Der Einfachheit halber werden nur die wesentlichen Befehle und Programmpakete in Klammer angeführt. Für die Signifikanz von Ergebnissen wurde generell ein Niveau von 0,05 herangezogen.

\subsection{Datenbestand}

Der für die Untersuchung zur Verfügung gestellte Datenbestand beinhaltet insgesamt 250.000 Datensätze im Sinne von Untersuchungsergebnissen für eine einzelne Probe. Der Umfang an Parametern für die einzelnen Proben variiert. Für die vorliegende Untersuchung sind relevant (Tab. 1).

Bei Vergleich zwischen den Parametern kann sich auch eine deutlich geringere Schnittmenge ergeben. Die Coliformen werden für eine einheitliche Untersuchung zusammengefasst. An zusätzlicher Information stehen im Wesentlichen Bezirk, Vorhandensein einer Desinfektionsanlage und der Typ der Probenahmestelle (PNS-Typ) zur Verfügung. Letzterer unterscheidet die folgenden Typen (Tab. 2).

Tab. 1 Anzahl Datensätze nach verschiedenen Parametern

\begin{tabular}{|l|l|}
\hline KBE bei $22^{\circ} \mathrm{C}[1 / \mathrm{ml}]$ & 190.000 \\
\hline KBE bei $37^{\circ} \mathrm{C}[1 / \mathrm{ml}]$ & 180.000 \\
\hline Coliforme $[100 / \mathrm{ml}]$ & 145.000 \\
\hline Coliforme $[250 / \mathrm{ml}]$ & 40.000 \\
\hline Temperatur $\left[{ }^{\circ} \mathrm{C}\right]$ & 220.000 \\
\hline TOC $[\mathrm{mg} / \mathrm{l}]$ & 32.000
\end{tabular}

Tab. 2 Anzahl Datensätze nach Typ der Probenahmestelle

\begin{tabular}{l|l}
\hline $\begin{array}{l}\text { Aufbereitung (UV-Anlage, } \\
\text { Nitratreduktion etc.) }\end{array}$ & 47.500 \\
\hline $\begin{array}{l}\text { Brunnen } \\
\text { Netz (Hausnetz, Ortsnetz) }\end{array}$ & 32.200 \\
\hline $\begin{array}{l}\text { Quelle } \\
\begin{array}{l}\text { Schacht (Übergabe-, Übernahme- } \\
\text { schacht) }\end{array}\end{array}$ & 117.000 \\
$\begin{array}{l}\text { Speicher (Tief-, Hochbehälter, } \\
\text { Quellsammelschacht) }\end{array}$ & 4400 \\
\hline
\end{tabular}


Nicht zuletzt aufgrund der Dominanz des PNS-Typs „Netz“ wird dieser vorrangig betrachtet. Der Zeitraum, für den Daten vorhanden sind, reicht im Wesentlichen von 1991 bis 2019, wobei die meisten Daten ab Beginn der 2000er-Jahre vorliegen.

Im Rahmen der Auswertungen der Temperaturdaten wurden auch meteorologische Daten der Lufttemperatur aus Wien (Stadt Wien o.J.) bzw. dem Flughafen Schwechat (rp5.co.uk o.J.) herangezogen.

\subsection{Trendanalyse}

Die Trendanalyse basiert auf der Darstellung und Regression der jährlichen Mittelwerte und 95-\%-Quantile im Fall der Temperatur sowie der jährlichen Mittelwerte und Anteil an Grenzwertüberschreitungen im Fall der mikrobiologischen Parameter. Der Grenzwert beträgt bei den $\mathrm{KBE}$ bei $22^{\circ} \mathrm{C} 100$ pro $\mathrm{ml}$, bei den $\mathrm{KBE}$ bei $37^{\circ} \mathrm{C} 20$ pro ml und bei den Coliformen 0 pro $100 \mathrm{ml}$ (die Grenzwerte für Proben unmittelbar nach Desinfektion wurden nicht berücksichtigt). Alle Kennzahlen wurden erst für jeden einzelnen Monat berechnet und anschließend durch Mittelwertbildung (arithmetisches Mittel) für das jeweilige Jahr zusammengefasst. Damit soll die unterschiedliche Probenanzahl im Jahresverlauf berücksichtigt werden. Jeder Monat geht mit dem gleichen Gewicht in den Jahreswert ein. Im Fall des 95-\%-Quantils bedeutet dies etwa eine Berechnung des Quantils für jeden einzelnen Monat und daraus die Mittelung für das jeweilige Jahr. Im Fall der Mittelwertbildung (nicht aber des Überschreitungsanteils) wurden hohe Werte entfernt und zwar alle Werte $\geq 300$ im Fall der KBE bzw. $\geq 50$ im Fall der Coliformen. Die gewählten Grenzwerte fallen im Fall der KBE mit typischen Grenzwerten der Auszählbarkeit für das Plattengussverfahren zusammen. Die Überlegung dabei ist gerade auch jene der Zurückdrängung des Einflusses einzelner, um Größenordnungen höherer Werte, wie sie bei biologischen Wachstumsprozessen auftreten können. Die Berechnung der Trends basiert auf der Anwendung einer linearen Regression („lm“, Paket „stats“) bzw. einer (ausreißerrobusten) linearen Regression (,rlm“, Paket „MASS“).

\subsection{Zeitreihenkorrelation}

Zur Beantwortung der Frage nach dem zeitlichen Versatz zwischen der Lufttemperatur und der Temperatur im Verteilnetz wurde in den jeweiligen Ganglinien die saisonale Komponente extrahiert („decompose“, Paket „stats“) und damit eine Kreuzkorrelation bei sukzessiver Verschiebung der Reihen gegeneinander berechnet („ccf“, Paket „stats“). Dadurch kann jene zeitliche Verschiebung gefunden werden, bei der die Zeitreihen die höchste Übereinstimmung zeigen.

\subsection{Modellansätze}

Zur Modellierung der Abhängigkeit der mikrobiologischen Parameter von anderen Parametern werden zwei Modellansätze gewählt, die im Folgenden kurz beschrieben werden. Die mikrobiologischen Parameter werden dabei grundsätzlich als Zähldaten aufgefasst, die im vorliegenden Fall häufig kleine Werte und einen hohen Anteil an NullWerten aufweisen (Francisque et al. 2009). Die weithin bekannte lineare Regression ist für diesen Fall weniger gut geeignet, da die Voraussetzung von normalverteilten Residuen (auch nach Transformation der Daten) nicht erfüllt werden kann (Fahrmeir et al. 2009).

\subsubsection{Logistische Regression}

Die logistische Regression dient der Modellierung diskreter abhängiger Variablen. Hier wird insbesondere die binomiale logistische Regression für binäre abhängige Variablen (zwei Ausprägungen) angewandt. $\mathrm{Zu}$ einer binären Ausprägung gelangt man, indem die abhängige Variable (mikrobiologischer Parameter) durch Vergleich mit dem jeweiligen Grenzwert laut Trinkwasserverordnung (KBE bei $22^{\circ} \mathrm{C}$ : $100, \mathrm{KBE}$ bei $37^{\circ} \mathrm{C}$ : 20 , Coliforme: 0) in Überschreitung bzw. Nicht-Überschreitung umgewandelt wird. Die Modellgleichung lautet (Fahrmeir et al. 2009):

$\log \left(\frac{P\left(y_{i}=1\right)}{1-P\left(y_{i}=1\right)}\right)=\beta_{0}+\beta_{1} x_{i 1}+\ldots+\beta_{k} x_{i k}$

Dabei können als unabhängige Variablen sowohl qualitative (Vorhandensein einer Desinfektionsanlage etc.) als auch quantitative Größen (Temperatur etc.) Eingang finden. Die Schätzung der Modellparameter in „ $\mathrm{R}^{\prime}$ erfolgt mit-
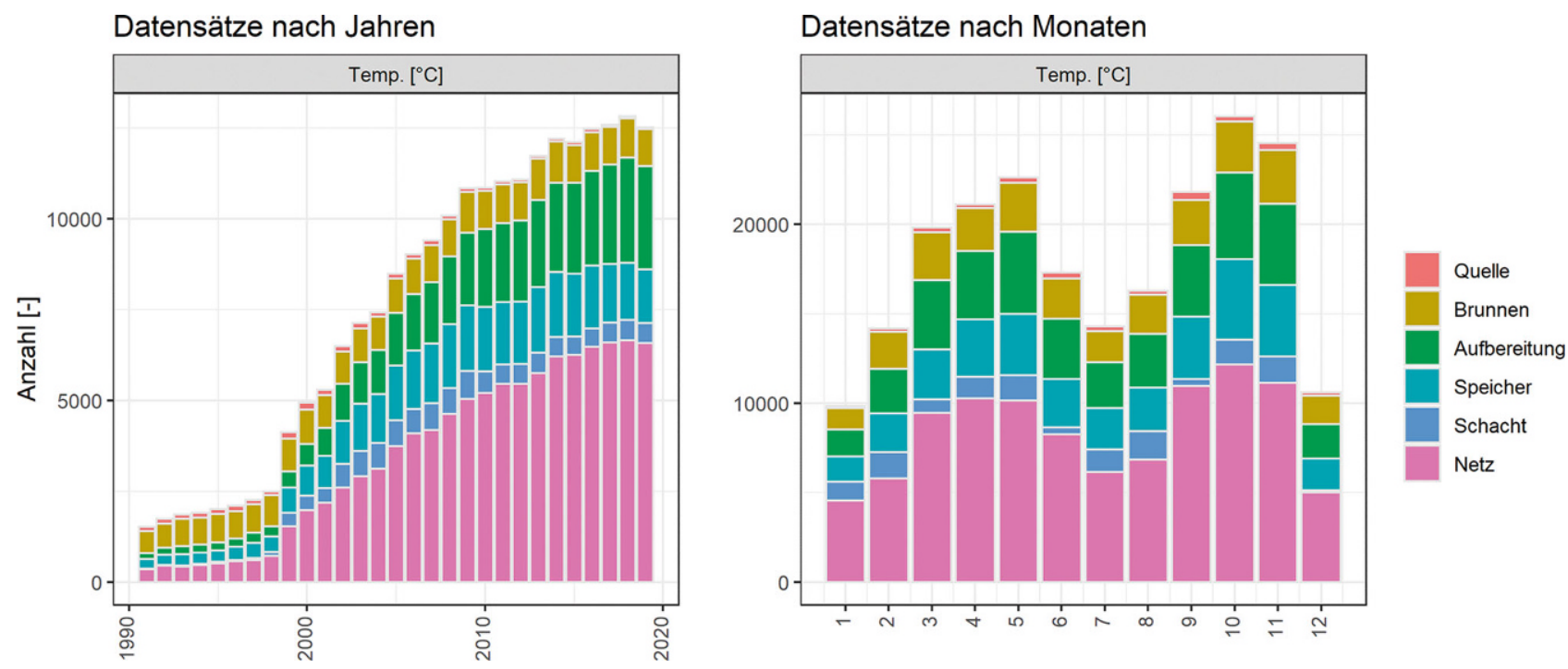

Abb. 1 Verfügbare Datensätze nach Jahren und Monaten gruppiert nach PNS-Typ 

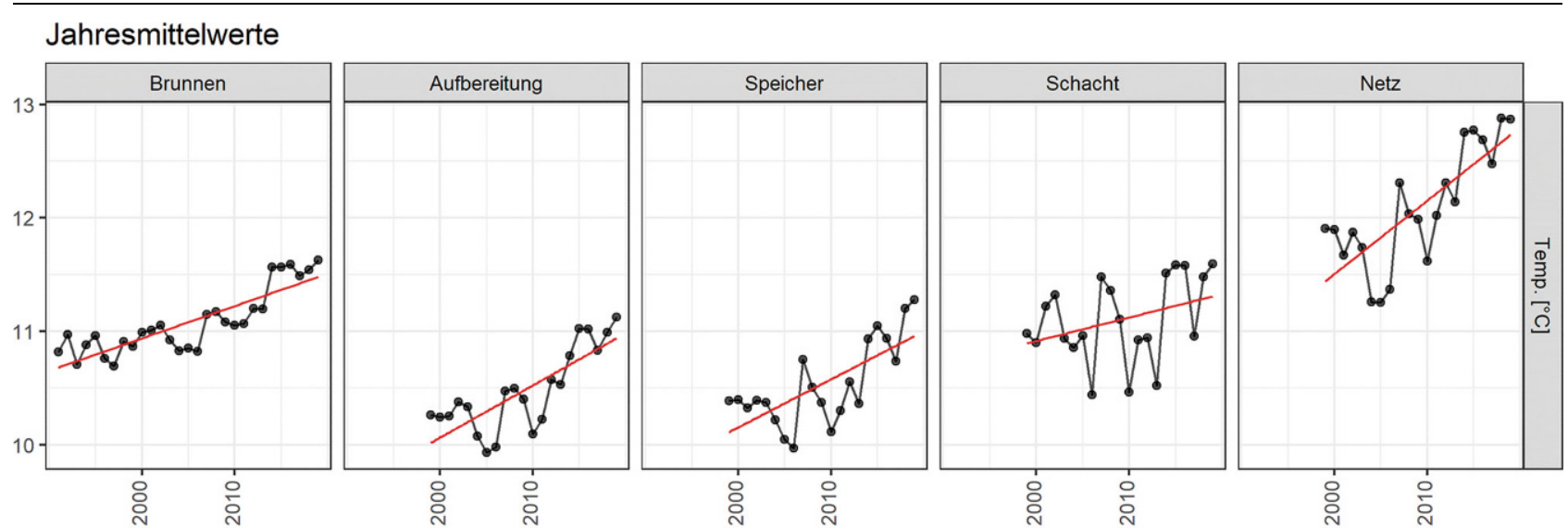

\section{Mittleres 95\%-Quantil}
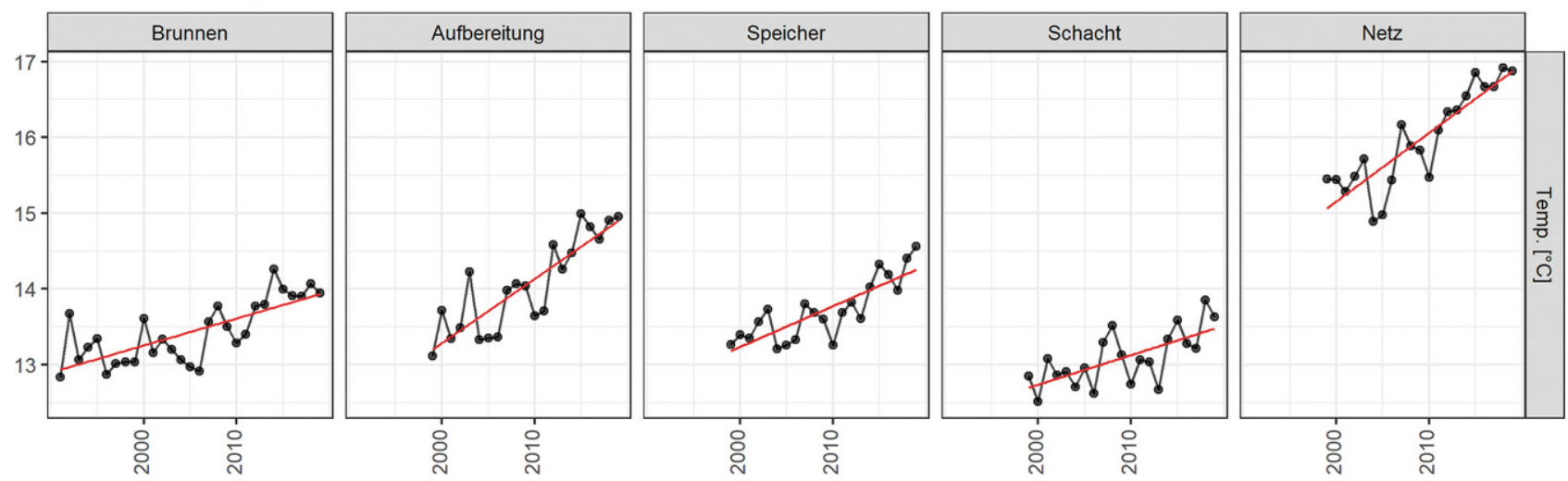

Abb. 2 Ganglinie der Jahresmittelwerte der monatlichen Mittelwerte und der monatlichen 95-\%-Quantile der Temperatur

tels der Funktion „glm“ aus dem Paket „stats“.

\subsubsection{Regression für Zähldaten}

Ein weiterer Ansatz bestand in der Anwendung von Modellen auf Basis der Poisson- bzw. negativen Binomialverteilung, auch als Regressionsmodelle für Zähldaten bezeichnet. Dabei werden im Unterschied zum ersten Ansatz die mikrobiologischen Werte (vereinfacht) nicht als binäres Ergebnis (Überschreitung und Nicht-Überschreitung) betrachtet, sondern als Zähldaten $(0,1$, $2, \ldots) . \mathrm{Zu}$ Vergleichszwecken wurden verschiedene Modellvarianten getestet, siehe dazu Gonzales-Barron et al. (2010) oder Francisque et al. (2009). Wie im Fall der Trendanalyse wurden auch hier einzelne sehr hohe Werte vor der Modellanwendung entfernt. In

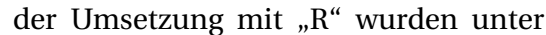
anderem die Funktionen „glm“ (Paket „stats“) und „glm.nb“ (Paket „MASS“) eingesetzt.

\section{Ergebnisse}

\subsection{Trinkwassertemperatur}

\subsubsection{Datenverfügbarkeit}

Für die Beurteilung der Temperaturentwicklung in verschiedenen Netzbereichen (PNS-Typ) stehen insgesamt etwa 220.000 Datensätze zur Verfügung. In Abb. 1 ist deren Verteilung über die Jahre 1991 bis 2019 getrennt nach PNS-Typ dargestellt. Aufgrund der guten Datenlage für die frühen Jahre wird für den Typ „Brunnen“ in den Auswertungen der gesamte Zeitraum herangezogen. Für die restlichen PNS-Typen erst Werte ab dem Jahr 1999. „Quellen“ werden aufgrund der geringen Datenverfügbarkeit insgesamt ausgeklammert.

Die Verteilung der Datensätze innerhalb eines Jahres zeigt Maxima im Frühjahr und im Herbst (für die Jahre 1991 bis 2019, rechts in Abb. 1 dargestellt), was bei bestimmten Auswertungen mitberücksichtigt werden muss. Insgesamt zeigen sich keine besonders augenscheinlichen Verzerrungen bei der Zusammensetzung der PNS-Typen über die Jahre sowie im Jahresverlauf.

\subsubsection{Trends}

Abb. 2 zeigt langjährige Verläufe der mittleren Jahrestemperatur und des 95\%-Quantils der Temperatur.

Es zeigt sich bei allen PNS-Typen ein positiver Trend. Der Trend bei den Mittelwerten ist bei allen PNS-Typen mit Ausnahme der Kategorie „Schacht“ signifikant. Die Zunahme für den PNSTyp „Netz“ beträgt hier im dargestellten Zeitraum $0,063^{\circ} \mathrm{C}$ pro Jahr (,rlm“) bzw. $0,065^{\circ} \mathrm{C}$ pro Jahr („Im“). Im Fall der 95-\%-Quantile, also der mittleren monatlichen Extremwerte, beträgt die Zunahme mit $0,085^{\circ} \mathrm{C}$ bzw. $0,091^{\circ} \mathrm{C}$ und ist ebenfalls signifikant.

In Abb. 3 ist ein Vergleich der Jahresmitteltemperatur für den PNS-Typ „Brunnen“ mit den Lufttemperaturen von Wien dargestellt (rechter Teil der Abbildung). Im linken Teil der Abbildung ist die Datenverfügbarkeit nach Jahr und Bezirk dargestellt, die keine besonderen Änderungen der Daten- 


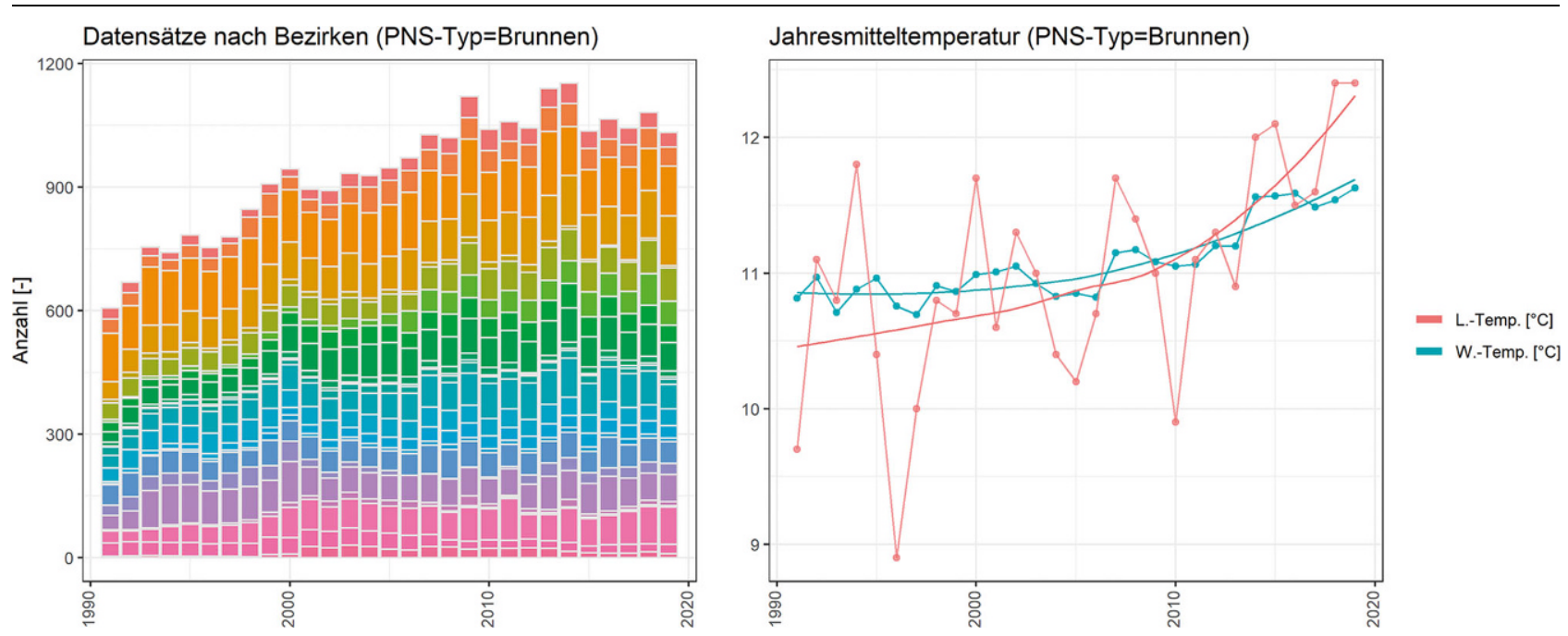

Abb. 3 Datenverfügbarkeit und Vergleich mit Lufttemperaturen (Wien) für den PNS-Typ „Brunnen“
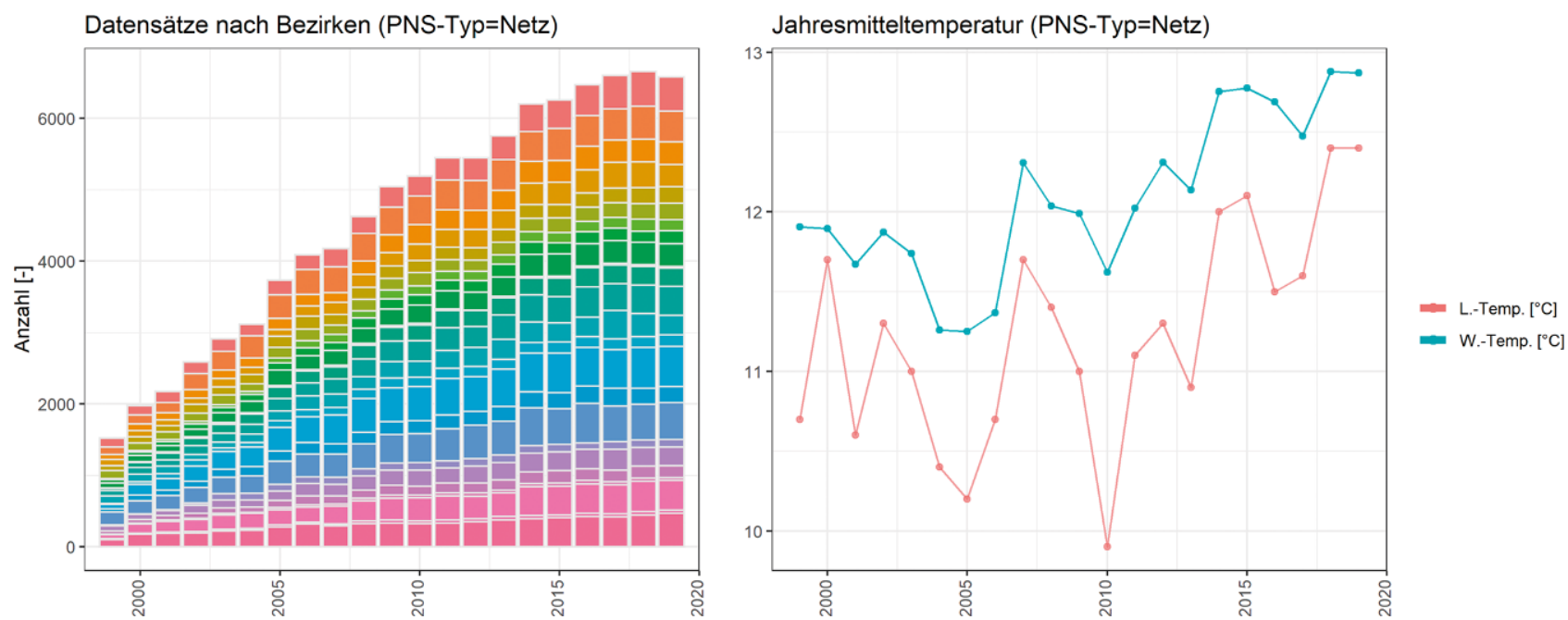

Abb. 4 Datenverfügbarkeit und Vergleich mit Lufttemperaturen (Wien) für den PNS-Typ „Netz“

zusammensetzung über die Jahre erkennen lässt. Es zeigt sich neben einer guten Übereinstimmung der Temperaturniveaus der Luft und in den Brunnen eine Zunahme-Tendenz der BrunnenWerte ab etwa Mitte der 2000er. Dabei erhöht sich der Mittelwert in den letzten 15 Jahren um gut $0,5^{\circ} \mathrm{C}$.

In Abb. 4 ist ein Vergleich der Jahresmitteltemperatur für den PNS-Typ "Netz“ mit den Lufttemperaturen von Wien dargestellt (rechter Teil der Abbildung). Im linken Teil der Abbildung ist die Datenverfügbarkeit nach Jahr und Bezirk dargestellt, die auch hier keine besonderen Änderungen der Datenzusammensetzung erkennen lässt. Im Unterschied zum PNS-Typ „Brunnen“ zeigt sich im "Netz“ ein sehr deutlicher Zusammenhang mit dem Verlauf der
Lufttemperatur (Ausnahme lediglich am Beginn der Zeitreihe).

Nicht nur im Mittel nehmen die Temperaturen zu, sondern auch die Extremwerte. In Abb. 5 ist der Anteil der Extremwerte $\left(\geq 20^{\circ} \mathrm{C}\right)$ für verschiedene Monate der Jahre 1999 bis 2019 dargestellt. Sowohl 2015 als auch 2018 wurden im Monat August Anteile über $30 \%$ verzeichnet, also annähernd jede dritte Probe des PNS-Typs „Netz“.

\subsubsection{Analyse des Zeitversatzes}

Die Lufttemperatur stellt einen sehr guten Kennwert für den Wärmehaushalt der bodennahen Atmosphäre dar und beschreibt damit auch die Dynamik der Bodenerwärmung. Aus dem Vergleich zwischen dem Verlauf der Lufttemperatur und der Temperatur im Leitungsnetz (hier auf Basis von Wochenmittelwerten) kann nun auf den zeitlichen Versatz zwischen beiden Ganglinien geschossen werden. Der Boden wirkt dabei als Puffer und so beeinflusst etwa eine Hitzeperiode erst mit zeitlicher Verzögerung die Temperatur im Leitungsnetz.

Für die Ganglinie der Temperatur im Leitungsnetz in Abb. 6 wurden aus den Temperaturdaten des ganzen Bundeslandes Wochenmittelwerte gebildet. Für die Lufttemperatur wurden Messdaten des Flughafens Wien herangezogen. Nach Entfernung des Trends in den Zeitreihen wurde eine saisonale Komponente angepasst, die die Grundlage für die Berechnung des Zeitversatzes bildet. Dieser beträgt 27 Tage, d.h., die Maxima oder Minima der Temperatur im Leitungsnetz treten im Mittel 

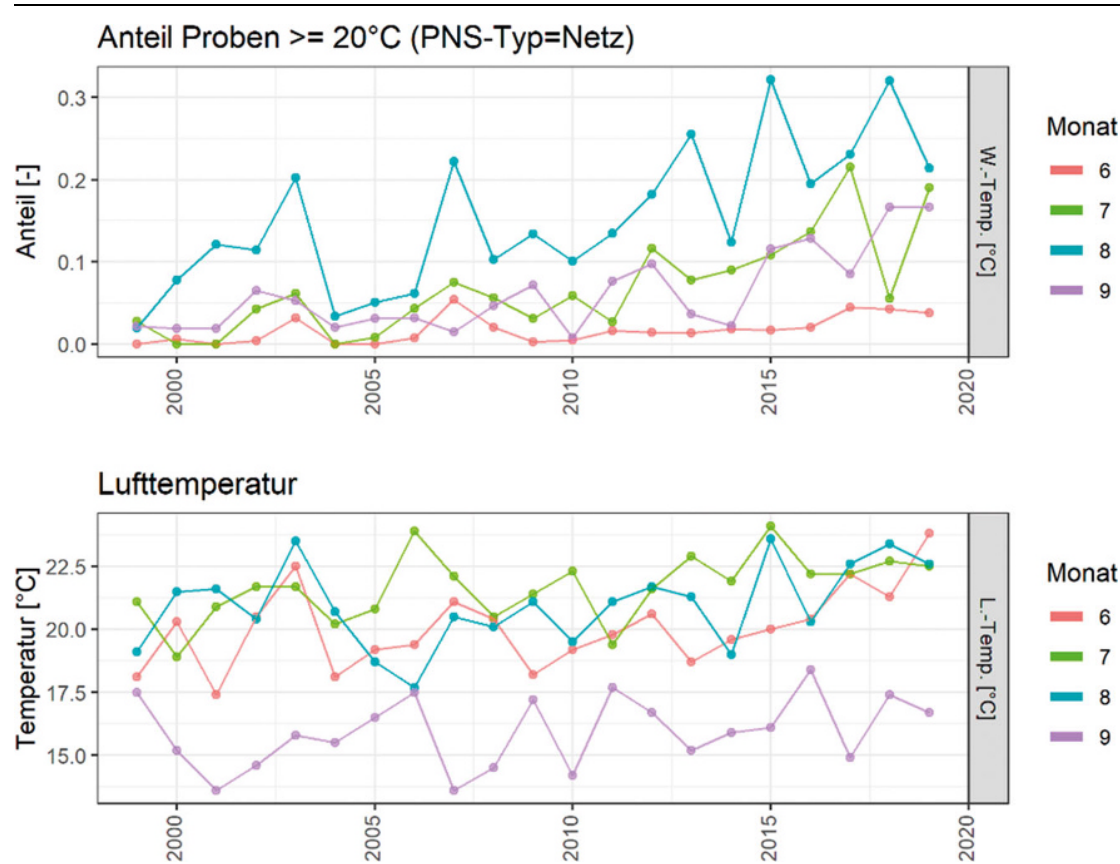

Abb. 5 Anteil der Proben mit einer Temperatur $\geq 20^{\circ} \mathrm{C}$ für die Monate Juni bis September

Ganglinie Wochenmitteltemperatur (PNS-Typ=Netz)

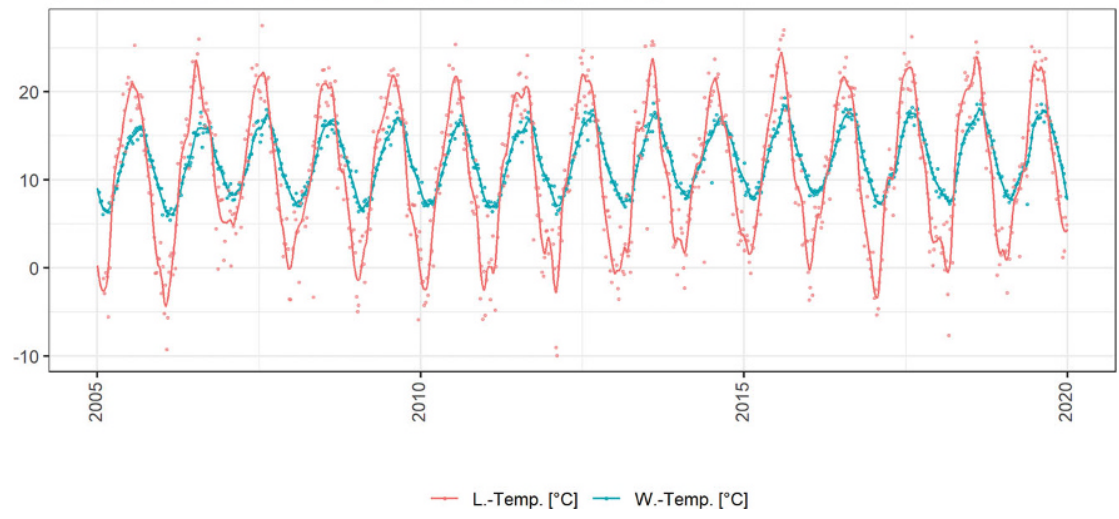

Abb. 6 Überlagerung der Ganglinien der Wochenmittel der Luft- und Netztemperatur

mit dieser Verzögerung gegenüber der Lufttemperatur auf. Geht man davon aus, dass die saisonale Komponente der Lufttemperatur durch die herangezogene Messstelle gut repräsentiert ist, sollte der ermittelte Zeitversatz die Verhältnisse im betreffenden Bundesland gut abbilden.

\subsection{Mikrobiologische Parameter mit Indikatorfunktion}

\subsubsection{Datenverfügbarkeit}

Für die Beurteilung des mikrobiologischen Zustands in verschiedenen Netzbereichen (PNS-Typ) stehen insgesamt etwa 550.000 Datensätze zur Verfügung,

\subsubsection{Trends}

Abb. 8 zeigt langjährige Verläufe des Mittelwerts und des Überschreitungsanteils der mikrobiologischen Parameter $\mathrm{KBE}$ bei $22^{\circ} \mathrm{C}$, KBE bei $37^{\circ} \mathrm{C}$ sowie Coliforme. Die Entfernung erhöhter Werte für die Auswertungen führen für den Parameter $\mathrm{KBE}$ bei $22^{\circ} \mathrm{C}$ zu einer Verringerung der Anzahl um etwa 2,5\%, im Fall der anderen Parameter sind weniger als $1 \%$ betroffen.

Im Gegensatz zu den Temperaturganglinien zeigen die mikrobiologischen Parameter häufig einen abnehmenden Trend. Für den PNS-Typ „Netz“ ist für die KBE ein signifikanter Rückgang des Mittelwerts und des Überschreitungsanteils zu verzeichnen. Der leicht zunehmende Trend bei den Coliformen ist nicht signifikant.

In Abb. 9 ist der mittlere monatliche Verlauf (Jahre 2005 bis 2019) der mikrobiologischen Parameter unterteilt nach PNS-Typen dargestellt. Bezüglich der Entfernung erhöhter Werte gelten die schon genannten Ausführungen. Die Verläufe in Abb. 9 lassen in vielen Fällen ein Minimum Ende Winter bzw. Anfang Frühling und Maxima in den Sommermonaten erkennen. Besonders augenscheinlich wird dies im Fall des PNS-Typs „Netz“, hier beträgt die Spanne zwischen Minimum (April) und Maximum (August) des Überschreitungsanteils der $\mathrm{KBE}$ bei $37^{\circ} \mathrm{C}(>20 \mathrm{KBE}$ pro $\mathrm{ml}$ ) über $5 \%$.

\subsubsection{Zusammenhang mit anderen Parametern}

In den folgenden Abschnitten wird der Einfluss einiger Parameter bzw. Parameterkombinationen auf die unterschiedlichen mikrobiologischen Parameter untersucht. Dabei kommen die beiden schon beschriebenen Modellansätze (Abschn. 2.4.) zur Anwendung. Temperatur In Abb. 10 ist das Ergebnis der Anpassung einer logistischen Regression für den Zusammenhang zwischen den $\mathrm{KBE}$ bei $37^{\circ} \mathrm{C}$ und der gemessenen Temperatur bei der Probenahme dargestellt. Der Einfluss der Temperatur ist dabei signifikant. So erhöht eine Zunahme der Temperatur von $15^{\circ} \mathrm{C}$ auf $20^{\circ} \mathrm{C}$ die Wahrscheinlichkeit von Werten $>20 \mathrm{KBE} / \mathrm{ml}$ von 0,077 auf 0,109 .

Im Fall der KBE bei $22^{\circ} \mathrm{C}(>100$ pro $\mathrm{ml}$ ) und der Coliformen ( $>0$ pro $100 \mathrm{ml}$ ) ist der Zusammenhang mit der Temperatur nicht signifikant. 
In Abb. 11 ist neben einem Histogramm der $\mathrm{KBE}$ bei $37^{\circ} \mathrm{C}$ (mit angepasster negativer Binomialverteilung für Werte <300) das Ergebnis der Anwendung einer Zähldaten-Regression in Abhängigkeit von der Temperatur dargestellt. Es besteht ein signifikanter positiver Zusammenhang.

Im Fall der KBE bei $22^{\circ} \mathrm{C}$ liegt ein positiver Zusammenhang vor, der in den meisten Modellansätzen signifikant ist. Der Effekt der Temperatur ist hier allerdings sehr gering und die vorhergesagte Zunahme beträgt lediglich $2 \mathrm{KBE} / \mathrm{ml}$ im Bereich zwischen $5^{\circ} \mathrm{C}$ und $25^{\circ} \mathrm{C}$. Im Fall der Coliformen liegt ein negativer Zusammenhang vor, der in einem der Modelle signifikant ist. Auch hier ist der Effekt der Temperatur als sehr gering zu bezeichnen.

Desinfektionsmaßnahmen Eine wesentliche Auswirkung auf den mikrobiologischen Zustand sollte das Vorhandensein einer Desinfektion im Zuge der Aufbereitung darstellen. Die Information über das Vorhandensein einer Desinfektionsanlage in der betreffenden Wasserversorgungsanlage ist Teil des Datensatzes und kann daher untersucht werden. Die Anzahl der Proben in beiden Gruppen (mit/ohne Desinfektion) ist für alle mikrobiologischen Parameter etwa gleich. Der Anteil an Überschreitungen von Grenzwerten ist bei Vorhandensein einer Desinfektion verringert (Jahre 2005 bis 2019). Für die KBE bei $22^{\circ} \mathrm{C}, 37^{\circ} \mathrm{C}$ sowie die Coliformen verringert sich der Anteil von 0,065 auf 0,050 , von 0,073 auf 0,062 sowie von 0,094 auf 0,065.

Bei Hinzunahme der Temperatur in die Modellgleichung der logistischen Regression (inklusive Interaktion) zeigt sich für die KBE bei $37^{\circ} \mathrm{C}$ (siehe Abb. 12) ein leicht modifiziertes Bild im Vergleich zu Abb. 10. Der Interaktionsterm bewirkt im Fall des Vorhandenseins einer Desinfektion eine etwas stärkere
Zunahme der Überschreitungswahrscheinlichkeit mit der Temperatur.

Total Organic Carbon Der Gehalt an organischer Substanz in einer Wasserprobe ist eine wesentliche Limitierung für die bakterielle Vermehrung (Prest et al. 2016). Wenngleich nur ein Bruchteil der gesamten Organik - ausgedrückt durch den TOC (Total Organic Carbon) - der bakteriellen Verwertung (unmittelbar) zugänglich ist, sollten hohe Werte tendenziell auch für eine hohe Nährstoffverfügbarkeit sprechen. Leider liegt der TOC im vorliegenden Datensatz in einem wesentlich geringeren Umfang vor wie etwa die mikrobiologischen Parameter $(N=16.500$ im Fall der Kombination mit mikrobiologischen Parametern).

Der TOC als einzige unabhängige Variable im Modell (logistische Regression) beeinflusst die Überschreitungen der KBE bei $22^{\circ} \mathrm{C}$ und $37^{\circ} \mathrm{C}$ im Sinne eines positiven Zusammenhangs. Bei

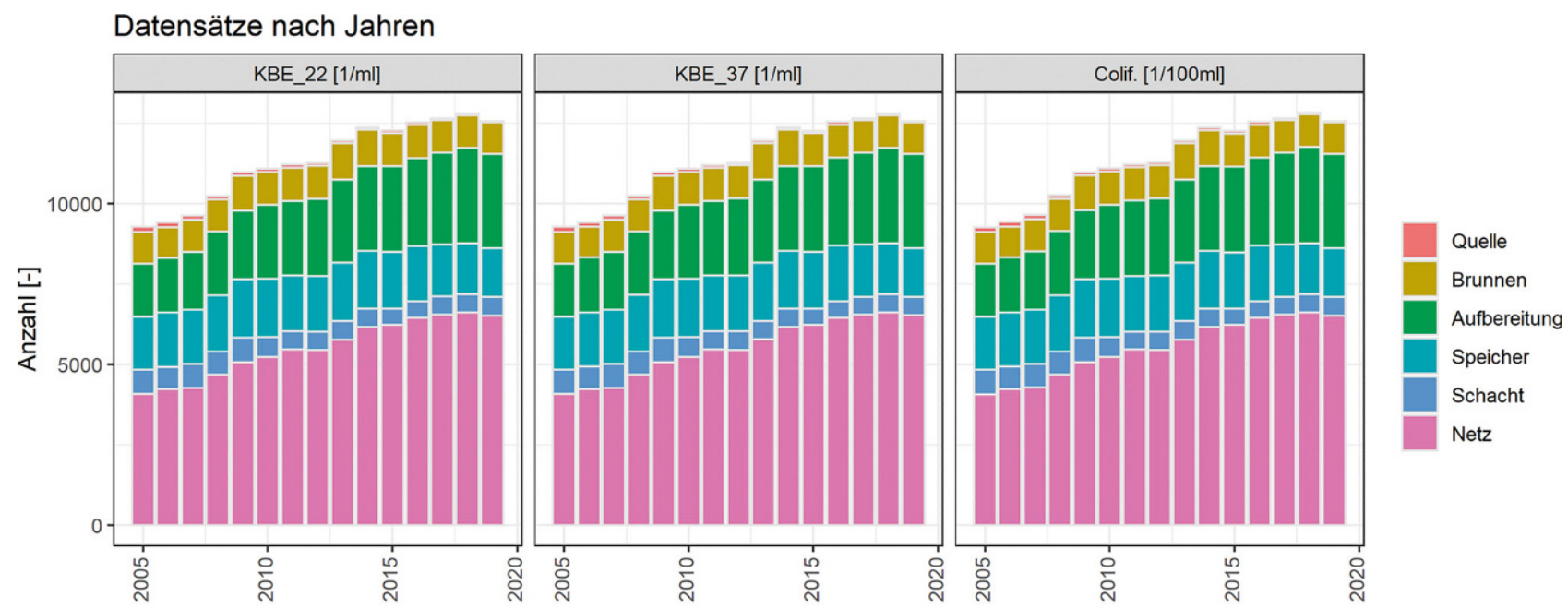

Datensätze nach Monaten
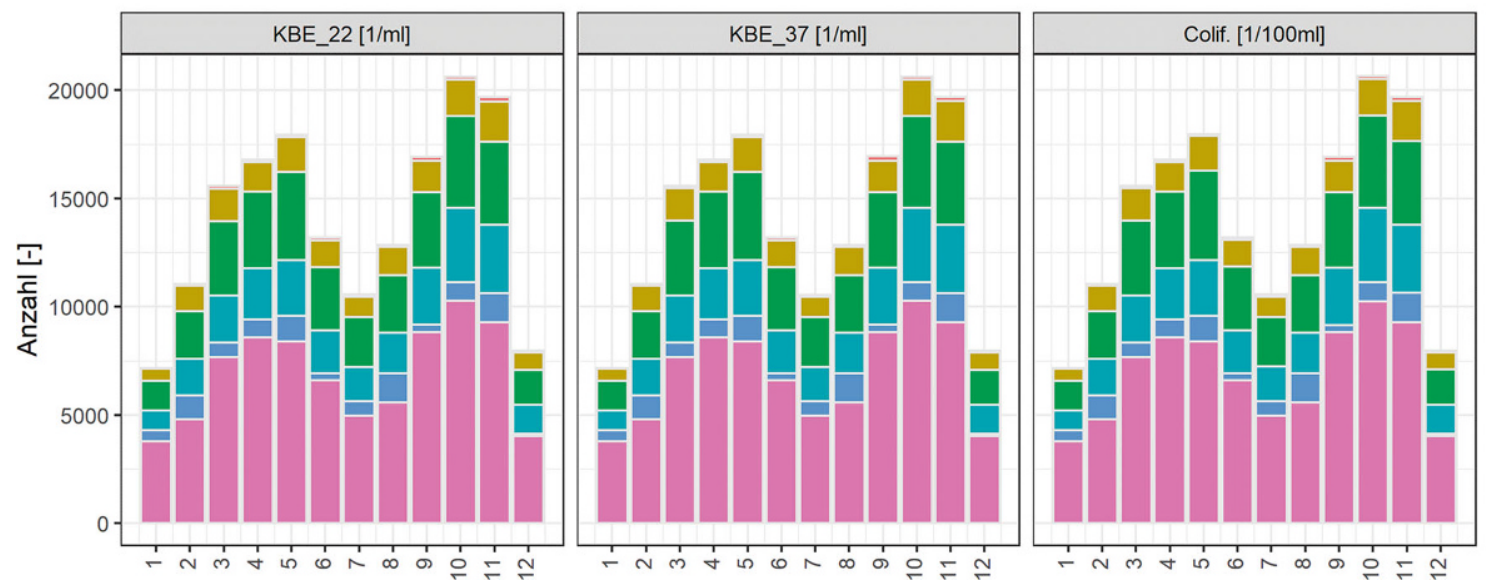

Quelle

Brunnen

Aufbereitung

Speicher

Schacht

Netz

Abb. 7 Verfügbare Datensätze der drei Parameter nach Jahren und Monaten sowie gruppiert nach PNS-Typ 


\section{Jahresmittelwerte}
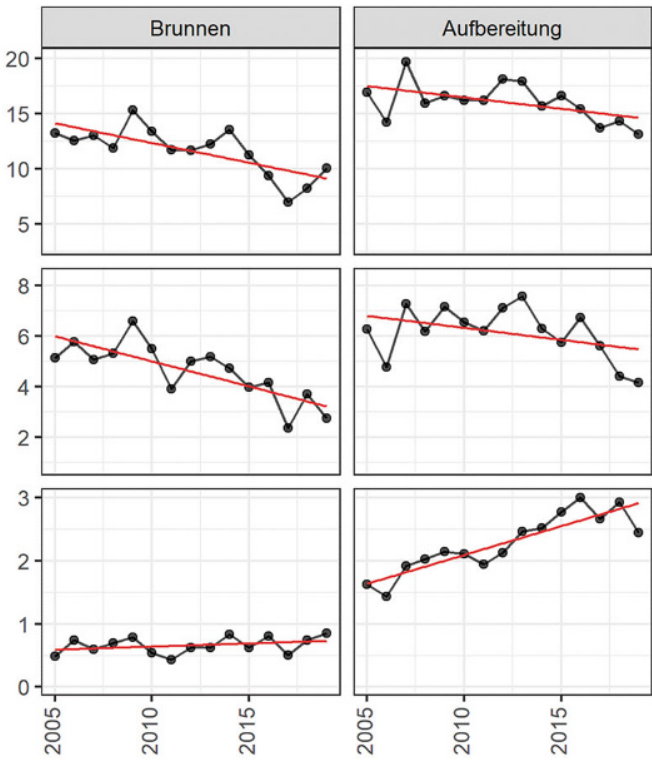

Mittlerer Überschreitungsanteil
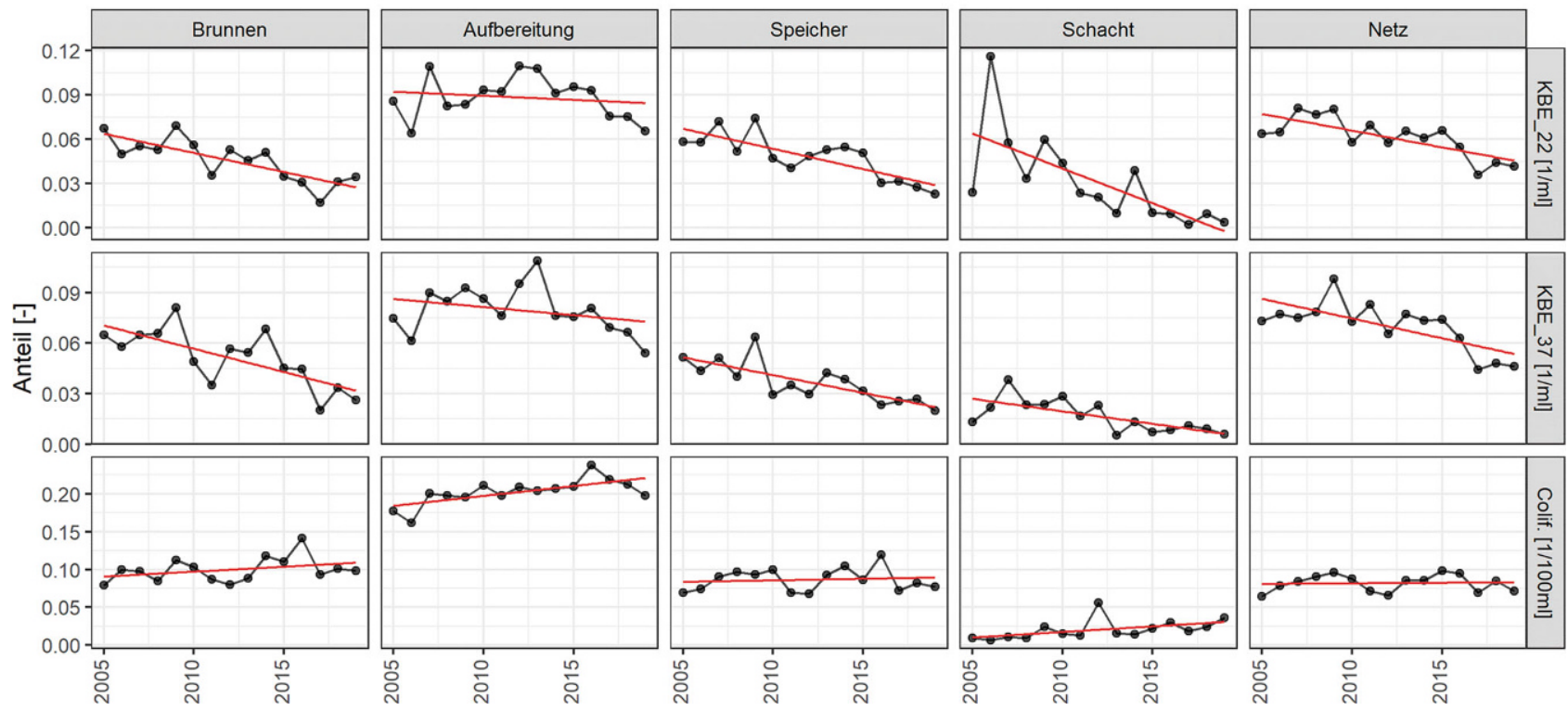

Abb. 8 Ganglinie der Jahresmittelwerte der monatlichen Mittelwerte und des monatlichen Überschreitungsanteils mikrobiologischer Parameter

den Coliformen ist dieser Einfluss nicht signifikant. In Abb. 13 ist das Ergebnis eines Modellansatzes für die KBE bei $37^{\circ} \mathrm{C}$ dargestellt, bei dem als Einflussfaktoren die Temperatur, der TOC sowie das Vorhandensein einer Desinfektion Eingang finden. Berücksichtigt werden zudem alle paarweisen Interaktionen. Nach schrittweiser Selektion („step“, Paket „stats“) verbleiben die Haupteffekte und die Interaktionsterme Desinfektion ${ }^{*}$ Temperatur (signifikant) und Desinfektion * TOC (nicht-signifikant) im Modell. Der TOC bewirkt auch hier eine Erhöhung der Wahrscheinlichkeit einer Überschreitung, wobei der Effekt bei Fehlen einer Desinfektion ausgeprägter ist.

Der Einfluss des TOC ist im Fall der Zähldaten-Regression für alle Parameter gegeben. Bei den Coliformen allerdings nur dann, wenn hohe Werte nicht entfernt werden.

\section{Diskussion und Ausblick}

\subsection{Temperatur}

Langfristige Trends der Temperaturen im Verteilnetz sind für einzelne Wasserversorgungen auf Basis der vorhandenen Untersuchungen nur sehr schwierig zu ermitteln. Gründe dafür sind die recht geringe Anzahl an Probenahmen, die ungleiche Verteilung der Probenahmen über das Jahr sowie Wechsel oder Ausweitung der Probenahmestellen. Die generelle, also bundeslandweite $\mathrm{Zu}$ - 


\section{Monatsmittelwerte}
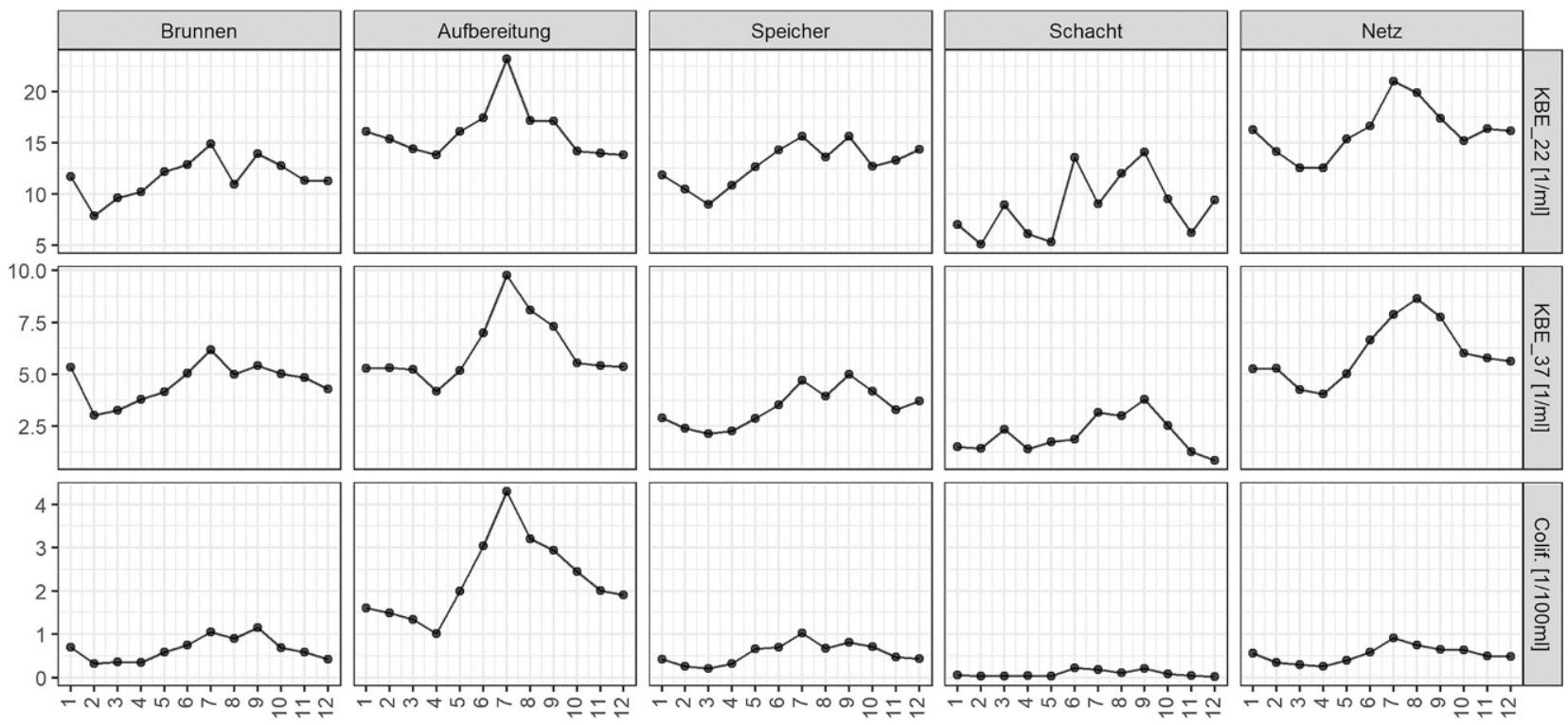

Mittlerer Überschreitungsanteil
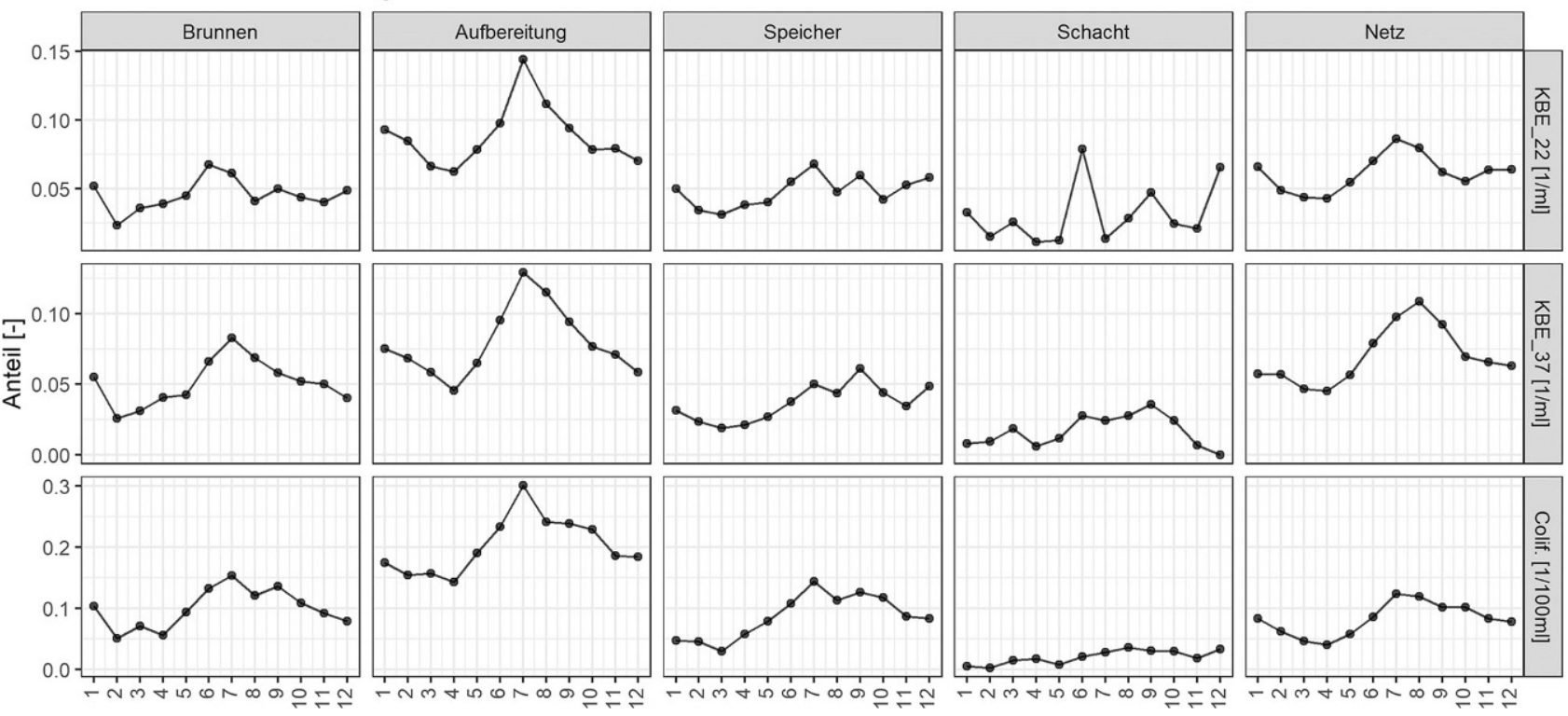

Abb. 9 Monatsmittelwerte mikrobiologischer Parameter basierend auf den monatlichen Mittelwerten und den monatlichen Überschreitungsanteilen der Jahre 2005 bis 2019

nahme-Tendenz sollte aber durch den vorliegenden Datensatz zumindest für die letzten 20 Jahre gut abgebildet sein und deckt sich auch mit den allgemeinen klimatischen Veränderungen. Eine systematische Verzerrung durch den Messvorgang selbst ist dabei weniger zu befürchten als etwa durch Änderungen von Verbrauchs- oder Betriebsmustern (Netzaufenthaltszeit, Entnahmemengen), bauliche Änderungen (thermische Situation) oder Änderungen bei der Zusammensetzung der Probenahmestellen (Ausweitung auf „extremere
Netzpunkte“). All diese Unsicherheiten ließen sich wohl nur durch langfristige und kontinuierliche Messprogramme unter Einbeziehung der Durchflussmengen und der Erfassung von Veränderungen im Untersuchungsgebiet beseitigen. Auch Simulationsstudien können hier eine Abschätzung der Situation bzw. Entwicklung liefern. Die Erhöhung von $0,64^{\circ} \mathrm{C}$ pro Jahrzehnt für den PNS-Typ „Netz“ sollte aber jedenfalls Beachtung finden.

\subsection{Mikrobiologische Parameter}

Eine interessante Beobachtung betrifft die vielfachen Abnahme-Tendenzen bei den mikrobiologischen Parametern. Die Ursachen dieser Abnahme sind nicht einfach $\mathrm{zu}$ eruieren. Eine Möglichkeit könnte in der vermehrten Installation von Desinfektionsanlagen bestehen. Allerdings gibt hier die getrennte Analyse von Versorgungen mit und ohne Desinfektion keinen Unterschied in den Trends hinsichtlich der KBE. Dagegen besteht eine gegensätz- 


\section{KBE_37 $>20$ (PNS-Typ=Netz)}

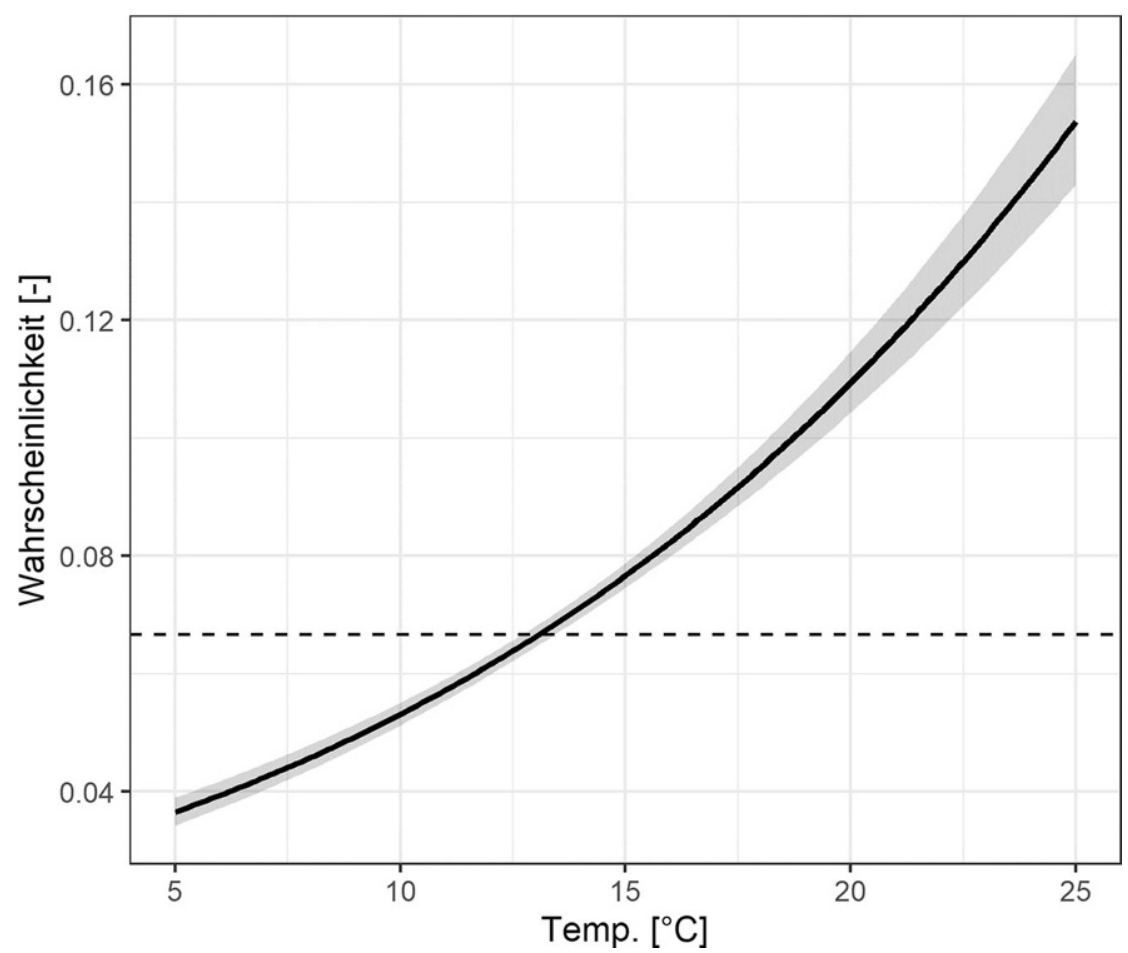

Abb. 10 Überschreitungswahrscheinlichkeit mit Konfidenzintervall (95\%) der KBE $37^{\circ} \mathrm{C}$ in Abhängigkeit von der Temperatur für den PNS-Typ „NNetz“. Die gestrichelte Linie kennzeichnet den Anteil an Überschreitungen im Datensatz

liche, wenngleich geringe Tendenz für die Coliformen - Abnahme bei Desinfektion, Zunahme ohne Desinfektion. Als vorschnell wäre jedenfalls die Schlussfolgerung $\mathrm{zu}$ bezeichnen, dass die Zunahme der Temperaturen da-

her keine (negativen) Auswirkungen auf die mikrobiologische Situation hat. Die angewandten Modelle ergeben hier eher, dass mit diesem Einflussfaktor $\mathrm{zu}$ rechnen ist, insbesondere im Hinblick auf die $\mathrm{KBE}$ bei $37^{\circ}$. Da letztere erhöhte Temperaturen zumindest bei der Kultivierung bevorzugen, liegt es auch nahe, dass erhöhte Wassertemperaturen zumindest keinen abträglichen Effekt haben. Zu betonen ist, dass ein statistischer Zusammenhang der Temperatur mit erhöhten Werten mikrobiologischer Parameter nicht zwingend kausal sein muss. Erhöhte Temperaturen können auch auf eine Stagnation des Wassers hindeuten. Die zeitgleich erhöhten Werte der untersuchten Parameter könnten demnach eher aus dem kombinierten Effekt eines anderen Einflussfaktors (etwa einer im Netz erhöhten Nährstoffverfügbarkeit) und längerer Aufenthaltszeit hervorgehen. In diesem Fall wäre die gemessene Temperatur eher als Indikator für die Aufenthaltszeit im Netz zu sehen.

\subsection{Ausblick}

Mit den genannten, auf Kultivierung basierenden Parametern wird nur ein kleiner Teil des mikrobiologischen $\mathrm{Zu}$ stands beleuchtet, da der überwiegende Teil der Bakterien im Wasser zumindest auf diese Art nicht kultivierbar ist. Technologische Entwicklungen der letzten Jahre ermöglichen die Erfassung mikrobiologischer Parameter, die andere Aspekte beschreiben und sich als sehr robust erwiesen haben (etwa die Totalzellzahl auf Basis der Durchflusszytometrie). Es wäre daher zumindest einer Überlegung wert, neuere methodische

\section{Grenzwert: 300}

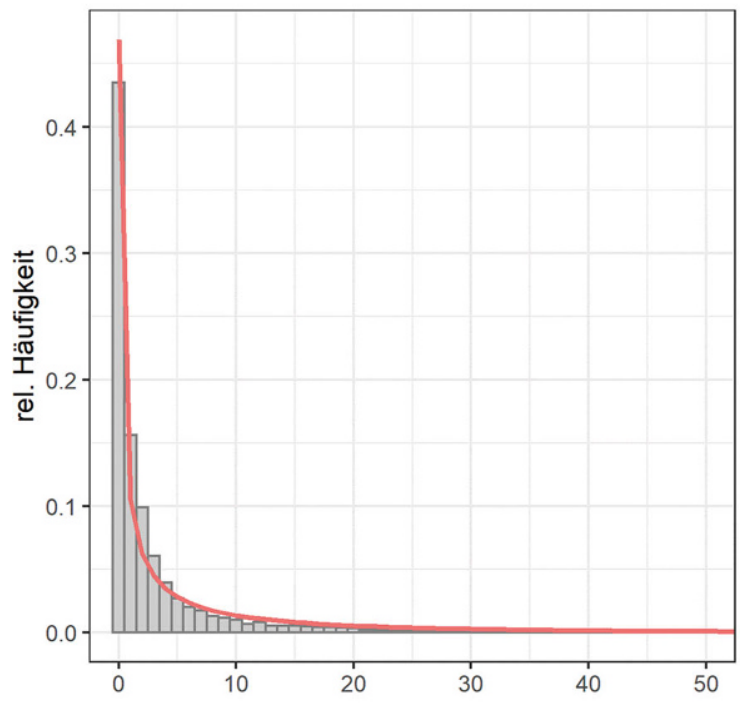

NegBin mit $\mathrm{Cl}-95 \%$

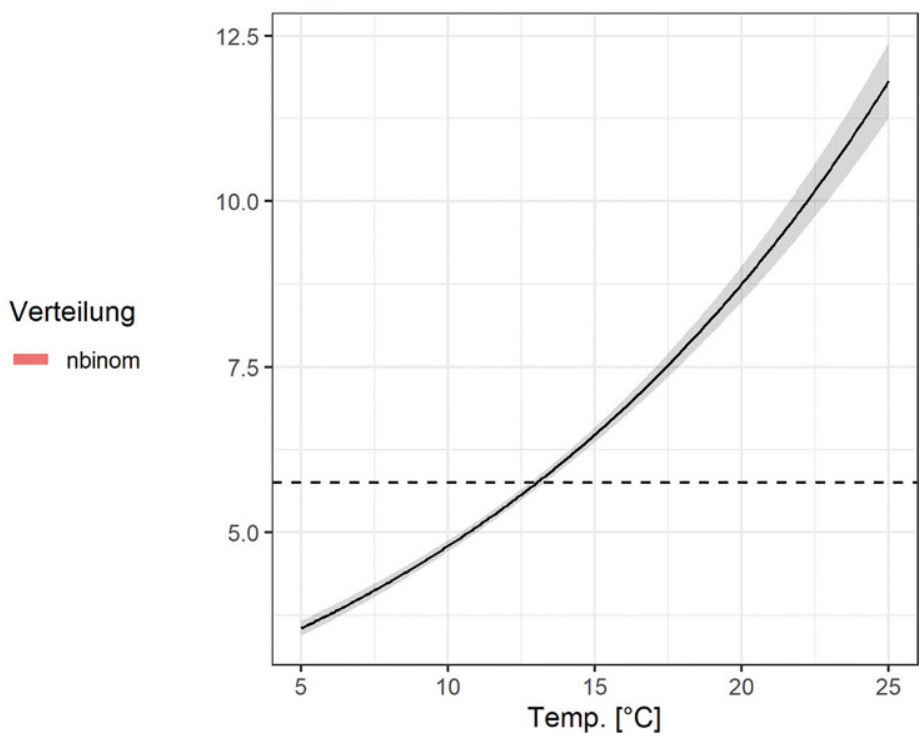

Abb. 11 Histogramm der KBE bei $37^{\circ} \mathrm{C}$ mit angepasster negativer Binomialverteilung (links) sowie Modellvorhersage in Abhängigkeit von der Temperatur für den PNS-Typ „Netz“(rechts). Die gestrichelte Linie kennzeichnet den Mittelwert des Datensatzes 


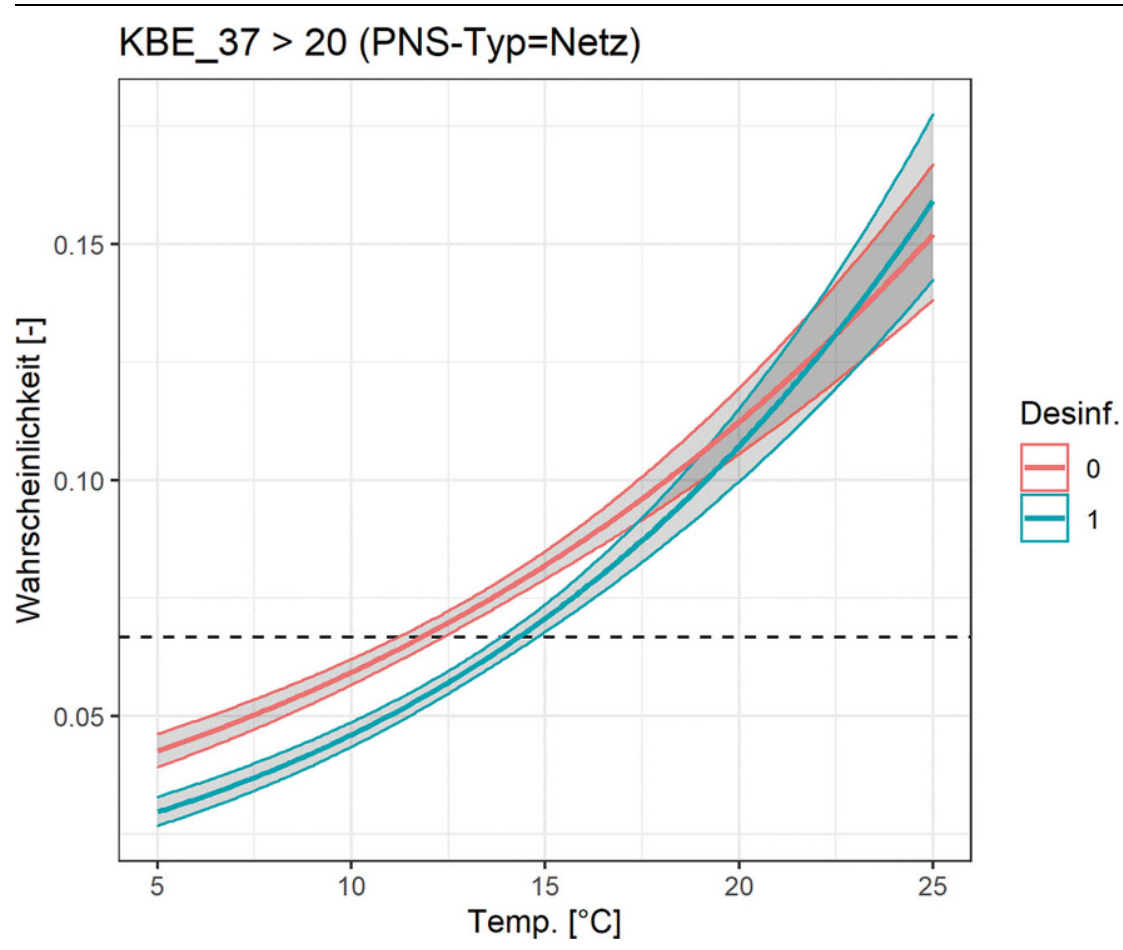

Abb. 12 Überschreitungswahrscheinlichkeit mit Konfidenzintervall (95\%) der KBE $37^{\circ} \mathrm{C}$ in Abhängigkeit von der Temperatur und dem Vorhandensein einer Desinfektion $\left(, 0^{\prime \prime}=\right.$ nein und „, “ = ja) für den PNS-Typ „Netz“. Die gestrichelte Linie kennzeichnet den Anteil an Überschreitungen im Datensatz
Ansätze in zukünftige Monitoringprogramme einfließen zu lassen.

Danksagung Ein besonderer Dank gilt allen Projektpartnern der Studie „Auswirkungen erhöhter Wassertemperaturen bei der Trinkwassergewinnung, -speicherung und -verteilung“, insbesondere dem Bundesministerium für Landwirtschaft, Regionen und Tourismus (BMLRT), der Österreichischen Vereinigung für das Gas- und Wasserfach (ÖVGW), den Ländern Niederösterreich, Oberösterreich, Salzburg und Steiermark sowie den beteiligten Wasserversorgern.

Funding Open access funding provided by University of Natural Resources and Life Sciences Vienna (BOKU).

Open Access Dieser Artikel wird unter der Creative Commons Namensnennung 4.0 International Lizenz veröffentlicht, welche die Nutzung, Vervielfältigung, Bearbeitung, Verbreitung und Wiedergabe in jeglichem Medium und Format erlaubt, sofern Sie den/die ursprünglichen Autor(en) und die Quelle ordnungsgemäß nennen, einen Link zur Creative Commons Lizenz beifügen und angeben, ob Änderungen vorgenommen wurden.

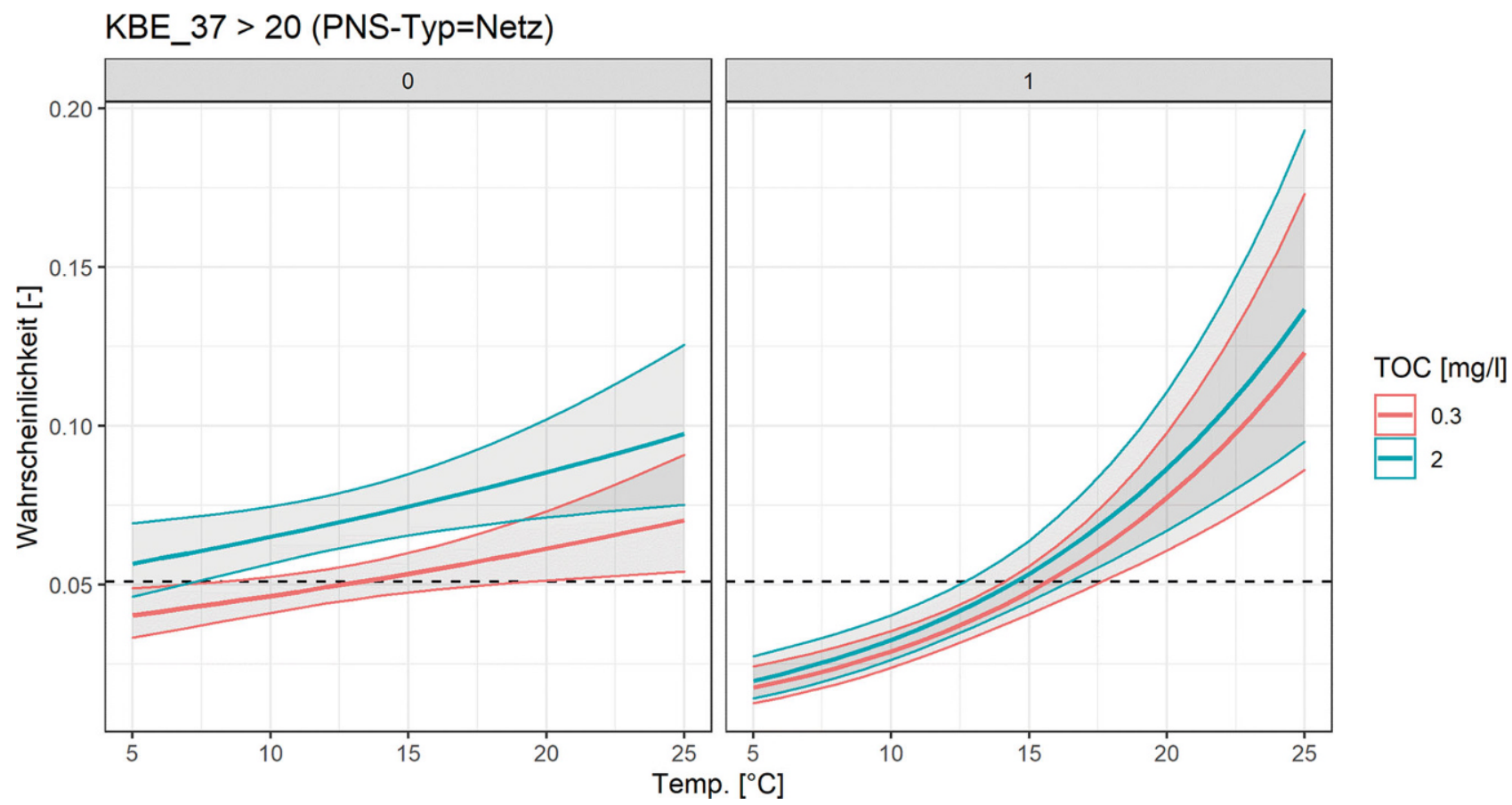

Abb. 13 Überschreitungswahrscheinlichkeit mit Konfidenzintervall (95\%) der Coliformen in Abhängigkeit von der Temperatur, dem TOC und dem Vorhandensein einer Desinfektion („,“ = nein und „1“ ” ja) für den PNS-Typ „Netz“. Die gestrichelte Linie kennzeichnet den Anteil an Überschreitungen im Datensatz 
Die in diesem Artikel enthaltenen Bilder und sonstiges Drittmaterial unterliegen ebenfalls der genannten Creative Commons Lizenz, sofern sich aus der Abbildungslegende nichts anderes ergibt. Sofern das betreffende Material nicht unter der genannten Creative
Commons Lizenz steht und die betreffende Handlung nicht nach gesetzlichen Vorschriften erlaubt ist, ist für die oben aufgeführten Weiterverwendungen des Materials die Einwilligung des jeweiligen Rechteinhabers einzuholen.
Weitere Details zur Lizenz entnehmen Sie bitte der Lizenzinformation auf http://creativecommons.org/licenses/ by/4.0/deed.de.

\section{Literatur}

Blöschl, G. und J. Parajka, A. P. Blaschke, M. Hofstätter, K. Haslinger, W. Schöner (2017): Klimawandel in der Wasserwirtschaft. Bundesministerium für Land- und Forstwirtschaft, Umwelt und Wasserwirtschaft, Wien

Fahrmeir, L. und T. Kneib, S. Lang (2009): Regression: Modelle, Methoden und Anwendungen. 2. Aufl. Springer

Feuerpfeil, I., und K. Botzenhart (2008): Hygienisch-mikrobiologische Wasseruntersuchung in der Praxis. Wiley-VCH, Weinheim

Francisque, A. und M. J. Rodriguez, L. F. Miranda-Moreno, R. Sadiq, F. Proulx (2009): Modeling of heterotrophic bacteria counts in a water distribution system. Water Research. https://doi org/10.1016/j.watres.2008.11.030

Gonzales-Barron, U. und M. Kerr, J. J. Sheridan,

F. Butler (2010): Count Data Distributions and Their Zero-Modified Equivalents as a Framework for Modelling Microbial Data with a Relatively High Occurrence of Zero Counts. International Journal of Food Microbiology. https://doi.org/ 10.1016/j.ijfoodmicro.2009.10.016

Grobe, S. und J. Wagner, J. Wingender (2014): Sicherung der Trinkwasserqualität bei der Wasserverteilung bei veränderten Bodentemperatu- ren. Die Veröffentlichung ist Teil der dynaklimProjekts (dynaklim-Publikation Nr. 52)

Prest, E. I. und F. Hammes, M. C. M. van Loosdrecht, J. S. Vrouwenvelder (2016): Biological Stability of Drinking Water: Controlling Factors, Methods, and Challenges. Frontiers in Microbiology. https://doi.org/10.3389/fmicb. 2016.00045

R Core Team (2019): R: A language and environment for statistical computing. R Foundation for Statistical Computing, Vienna, Austria. URL https://www.R-project.org/

Riedel, T. (2019): Temperature-Associated Changes in Groundwater Quality. Journal of Hydrology. https://doi.org/10.1016/j.jhydrol.2019.02. 059

rp5.co.uk (o. J.): Wetterarchiv in Wien (Flughafen). URL http://rp5.co.uk/Wetterarchiv_in Wien_Flughafen). 07.08.2020

Stadt Wien (o. J.): Lufttemperatur in Wien 1955 bis 2019. URL https://www.wien.gv.at/statistik/ lebensraum/tabellen/temperatur-zr.html. Zugriffsdatum: 07.08.2020

UBA (Deutsches Umweltbundesamt) (2009): Coliforme Bakterien im Trinkwasser: Empfehlung zur Risikoabschätzung und Maßnahmen bei systemischer Kontamination - Empfehlung des Umweltbundesamtes nach Anhörung der Trinkwasserkommission des Bundesministeriums für Gesundheit beim Umweltbundesamt. Bundesgesundheitsblatt - Gesundheitsforschung - Gesundheitsschutz. https://doi.org/10.1007/ s00103-009-0823-7

Van Nevel, S. und S. Koetzsch, C. R. Proctor, M. D. Besmer, E. I. Prest, J. S. Vrouwenvelder, A. Knezev, N. Boon, F. Hammes (2017): Flow cytometric bacterial cell counts challenge conventional heterotrophic plate counts for routine microbiological drinking water monitoring. $\mathrm{Wa}$ ter Research. https://doi.org/10.1016/j.watres. 2017.01.065

Hinweis des Verlags Der Verlag bleibt in Hinblick auf geografische Zuordnungen und Gebietsbezeichnungen in veröffentlichten Karten und Institutsadressen neutral. 